\title{
Effect of Circular ANRIL on the Inflammatory Response of Vascular Endothelial Cells in a Rat Model of Coronary Atherosclerosis
}

\author{
Chun-Li Song Jin-Peng Wang Xin Xue Ning Liu Xiao-Hao Zhang Zhuo Zhao \\ Jian-Gen Liu Chun-Peng Zhang Zhe-Hao Piao Yang Liu Yi-Bo Yang \\ Department of Cardiovascular Internal Medicine, The Second Hospital of Jilin University, Changchun, \\ P.R. China
}

\section{Key Words}

Circular RNA ANRIL • Coronary atherosclerosis • Endothelial cells • Inflammatory response • Apoptosis

\begin{abstract}
Background/Aims: This study aims to investigate the role of circular antisense non-coding RNA at the INK4 locus (CANRIL) in the inflammatory response of vascular endothelial cells (ECs) in a rat model of coronary atherosclerosis (AS). A rat model of AS was established with rats that were injected with a large dose of vitamin $D_{3}$ and fed a high-fat diet. Methods: Sixty Wistar rats were randomly assigned into control, model, empty vector, over-expressed cANRIL and low-expressed cANRIL groups (12 rats in each group). Sixteen weeks later, the ultrastructure of their coronary arteries was observed via transmission electron microscopy. Rat serum lipid levels were analyzed using an automatic biochemical analyzer, and their atherogenic index (AI) values were calculated. Hematoxylin and eosin staining was used to observe the endothelial morphology of rats. Additionally, rat EC apoptosis was tested via a TUNEL assay. Enzyme-linked immunosorbent assays (ELISAs) were applied to measure serum levels of interleukin-1 (IL-1), IL-6, matrix metalloproteinase-9 (MMP-9) and C-reactive protein (CRP). The CANRIL, Bax, bcl-2 and caspase-3 mRNA expression levels were measured with a quantitative real-time polymerase chain reaction (qRT-PCR). The protein expression levels of Bax, bcl-2 and caspase-3 were detected using immunohistochemistry. Results: In the control group, ECs were closely arranged with normal structures, and there was no proliferation. In the model, empty vector and over-expressed CANRIL groups, some cells were not present, and atherosclerotic plaques and thrombi appeared. However, in the under-expressed CANRIL group, the cells had a normal structure. Compared with the model and empty vector groups, the levels of total cholesterol (CHOL), triglycerides (TGs), low density lipoprotein (LDL), IL-1, IL-6, MMP-9, CRP, CANRIL, Bax, and caspase-3, AI values, and rates of EC apoptosis decreased in the low-expressed cANRIL group, while HDL (high density lipoprotein) levels and mRNA and protein expression levels of bcl-2 were increased. The changes in expression levels in the
\end{abstract}


over-expressed CANRIL group were the opposite of those in the low-expressed cANRIL group. Conclusions: Our study provides evidence that reduced cANRIL expression could prevent coronary AS by reducing vascular EC apoptosis and inflammatory factor expression.

(C) 2017 The Author(s)

Published by S. Karger AG, Basel

\section{Introduction}

Atherosclerosis (AS) is a chronic disease of arterial walls and a main cause of loss of productive lifespan and death worldwide [1,2]. AS is also affected by several factors, such as gender differences (more frequent in males), hypertension, family history [3], and a decades-long expansion between the underlying smooth muscle cells (SMCs) of the tunica media and endothelium [4]. The structural and functional integrity of the endothelium is crucial in maintaining cardiovascular homoeostasis and preventing AS [5]. Cardiovascular research suggests that endothelial dysfunction is the initial step in the development of AS [6]. Dysfunction of the arterial vasculature is a main contributor to atherosclerotic cardiovascular disease and the initiation and progression of AS $[7,8]$.

ANRIL encodes a long non-coding RNA (lncRNA) at the INK4 locus that overlaps with the 9 p21.3 risk interval. It plays distinct regulatory roles in the expression of adjacent proteincoding genes, including methylthioadenosine phosphorylase (MTAP), and 2 cyclin-dependent kinase inhibitors, CDKN2A (encoding p16 ${ }^{\mathrm{INK} 4 \mathrm{a}}$ and p14 ${ }^{\mathrm{ARF}}$ ) and CDKN2B (encoding p1 $5^{\mathrm{INK} 4 \mathrm{~b}}$ ) [9]. ANRIL gene expression has been documented in atheromatous vessels, coronary SMCs and vascular endothelial cells (ECs) [10]. The lncRNA ANRIL participates in the replication of the genetic risk locus for coronary artery disease (CAD), and it is independently correlated with several forms of immune-mediated, metabolic disorders and coronary heart disease $[11,12]$. Previous work has demonstrated that the lncRNA ANRIL, which is transcribed at the cardiovascular disease risk locus on chromosome 9p21, can form RNA circles [13]. Circular RNAs (cRNAs) are a special type of a stable, diverse and conserved noncoding RNAs in mammalian cells [14]. In addition, cRNAs widely participate in the physiological/pathological processes involved in cancer [15]. Wu et al. reported that the different expression patterns of circulatory cRNAs during heart failure (HF) suggest that cRNAs may actively respond to stress and thus serve as indicators for diagnosing HF [16]. As a type of cRNA, cANRIL can inhibit pre-ribosomal RNA processing mediated by exonuclease and prohibit ribosome biogenesis and the proliferation of cells, which may contribute to AS by inducing apoptosis in vascular SMCs [17]. However, there are insufficient data to demonstrate the involvement of cANRIL in the inflammatory response of vascular ECs during the development of AS. Here, we explored how cANRIL regulates the inflammatory response of vascular ECs in association with AS with the aim of developing new avenues for preventing and treating AS.

\section{Materials and Methods}

\section{Experimental animals and grouping}

Sixty clean-grade healthy male Wistar rats were provided by the Experimental Animal Center of Third Military Medical University. Each rat weighed 200 240 grams and was 8 weeks old. Rats were housed in standard animal laboratories with a controlled temperature of $23^{\circ} \mathrm{C}$, a humidity of $50 \%$, an artificial 12 $\mathrm{h}$ light-dark cycle and free access to food and water. Rats were maintained under these conditions for 1 week to adapt to the environment and were then randomly divided into the following five groups: control group, model group, empty vector group (injected with empty cANRIL vectors through the caudal vein after model establishment), over-expressed cANRIL group (injected with cANRIL mimics through the caudal vein after model establishment) and low-expressed cANRIL group (injected with cANRIL inhibitors through the caudal vein after model establishment). There were 12 rats in each group, and all groups, except the control group, served as the experimental model. Rats in the control group were fed a normal diet and were injected with physiological saline through the abdominal cavity once a month. In the empty vector, over-expressed cANRIL, low-expressed cANRIL and model groups, each rat was injected with vitamin $\mathrm{D}_{3}$ 


\section{Cellular Physiology Cell Physiol Biochem 2017;42:1202-1212

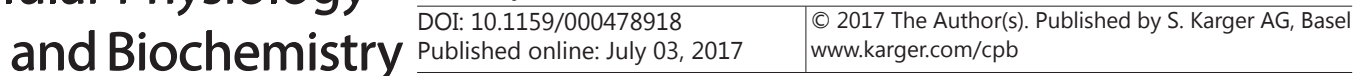 \\ Song et al.: Role of cANRIL in Atherosclerosis}

(Shanghai General Pharmaceutical Co., LTD., Shanghai, China) at a total dose of 70,000 U/kg over 3 days. Then, the rats were fed a high-fat diet containing $4 \%$ cholesterol (CHOL), 5\% sugar, 10\% lard and $82 \%$ of the normal diet for 21 days. Then, $10 \mu \mathrm{L}$ of empty vector, cANRIL mimics and cANRIL inhibitors (all provided by Guangzhou Geneseed Biotech Co., Ltd. Guangdong, China) was injected into the rats in the corresponding groups through the caudal vein. After an injection, the needle was kept in the vein for $1 \mathrm{~min}$, and bleeding was stopped with a tampon; then, the rats were maintained on a high-fat diet. All rats were killed at week 16. Experimental procedures were performed in accordance with the ethical guidelines for the study of experimental pain in conscious animals.

\section{Sample collection}

After they were injected with vectors and fed for 16 weeks, $1.5 \mathrm{~mL}$ of blood was collected from the retro-orbital venous plexus from each rat in each group. After centrifugation at $3000 \mathrm{rpm}$ for $15 \mathrm{~min}$, the serum was collected and frozen at $-80^{\circ} \mathrm{C}$. Rats were deeply anesthetized with pentobarbital $(40 \mathrm{mg} / \mathrm{kg}$, purchased from Sigma-Aldrich Chemical Company, St Louis MO, USA) and held in place on a fixation plate. Their hearts were quickly removed and then pressed until the residual blood was discharged. An incision was made along the bottom $1 / 4$ of the right ventricle, and the right coronary artery was identified relative to the root of the aorta. The left ventricle was elevated, and the left anterior descending coronary artery was identified based on the root of the aorta. This allowed a complete segment of coronary artery tissue to be obtained (including the right coronary artery and left anterior descending coronary artery) and fixed in 4\% glutaraldehyde (purchased from Sigma-Aldrich Chemical Company, St Louis MO, USA), while another section of the coronary artery was placed in $10 \%$ polyformaldehyde (Sigma-Aldrich Chemical Company, St Louis MO, USA). Then, paraffin-embedded specimens were produced and preserved at a low temperature. In addition, coronary artery tissue samples were digested in $0.1 \%$ type II collagenase for $15 \mathrm{~min}$, and the liquid was collected and centrifuged at $1200 \mathrm{rpm}$ for $8 \mathrm{~min}$; then, the supernatant was discarded and coronary artery ECs were collected and preserved at $-80^{\circ} \mathrm{C}$.

\section{Determination of serum lipids and the atherogenic index (AI)}

After rats were injected with vectors and fed for 16 weeks, $1 \mathrm{~mL}$ of serum was collected from each rat, and total CHOL, triglyceride (TG), low-density lipoprotein (LDL) and high-density lipoprotein (HDL) levels were evaluated using an automatic biochemical analyzer. Then, the AI was calculated as follows: AI = (CHOL-HDL)/HDL.

\section{Transmission electron microscopy (TEM)}

Complete coronary artery tissue samples were fixed in $4 \%$ glutaraldehyde for more than $2 \mathrm{~h}$ and washed in phosphate-buffered saline (PBS) for $5 \mathrm{~min}$, which was repeated 3 times. Then, the cells were fixed in 1\% osmic acid for 1-2 $\mathrm{h}$ and washed with PBS for $5 \mathrm{~min}$. The procedure was repeated 3 times and was followed by dehydration using 50\%, 70\%, 90\% and 100\% acetone for 15 min and infiltration with embedding mediums of 2: 1 acetone and 1: 1 acetone for $2 \mathrm{~h}$. Subsequently, the tissue blocks were embedded in a rubber tile with wells and warmed at $37^{\circ} \mathrm{C}, 45^{\circ} \mathrm{C}$, and $60^{\circ} \mathrm{C}$ for $12 \mathrm{~h}$ to produce specimens for analysis. The ultrastructure of the coronary artery was observed via TEM (TECNAIG2, Philips, Eindhoven, Netherlands).

\section{Hematoxylin and eosin (H\&E) staining}

Coronary artery specimens from each group were fixed in $10 \%$ formalin (Nanjing Jiancheng Bioengineering Institute, Nanjing, China) for $24 \mathrm{~h}$ and then dehydrated in ethanol, cleared in xylene, deparaffinized and embedded in paraffin. Afterwards, $4-\mu$ m-thick paraffin sections were produced. After deparaffinization, the specimens were stained with hematoxylin for $5 \mathrm{~min}$, differentiated in $1 \%$ acid alcohol, stained with eosin for $3 \mathrm{~min}$, dehydrated in ethanol and cleared in xylene twice for $10 \mathrm{~min}$. The specimens were then dripped with gum arabic and covered with coverslips. The morphological changes in the coronary arteries were observed using optical microscopy. Xylene, ethanol, hematoxylin, eosin and gum arabic were provided by Sigma-Aldrich Chemical Company (St Louis, MO, USA). The LM1235 Ultra-Thin Semiautomatic Microtome was purchased from Leica Company (Germany). The KD-BM machine used for paraffin embedding was purchased from Jinhua Kolno Electronic Technology Co., Ltd. (Zhejiang province, China). The CX-31 optical microscope was provided by Olympus Optical Co., Ltd. (Tokyo, Japan). 
Terminal deoxyribonucleotide transferase (TDT)-mediated dUTP-digoxigenin nick end labeling (TUNEL) assay

EC apoptosis in coronary arteries was measured using a TUNEL assay kit (purchased from Promega Corp., Madison, Wisconsin, USA) based on the manufacturer's instructions. Coronary artery samples were paraffin embedded and sectioned ( $5 \mu \mathrm{m}$ thick) to produce specimens for analysis. The specimens underwent deparaffinization heating for $3 \mathrm{~h}$ and were then treated with protease $\mathrm{K}$ for $30 \mathrm{~min}$ at $37^{\circ} \mathrm{C}$, which was followed by soaking in $3 \% \mathrm{H}_{2} \mathrm{O}_{2}$ and sealing at room temperature for $10 \mathrm{~min}$. Then, the specimens were treated with $50 \mu \mathrm{L}$ of TDT and reacted in the dark for $60 \mathrm{~min}$. Afterwards, $50 \mu \mathrm{L}$ of streptavidin-HRP was added, and the solution was reacted in the dark for $30 \mathrm{~min}$. Finally, the specimens were treated with 50-100 $\mu \mathrm{L}$ of diaminobenzidine (DAB) and reacted at room temperature for $10 \mathrm{~min}$. Between every 2 steps of the procedure, the specimens were washed in PBS for $2 \mathrm{~min}$, which was repeated 3 times. The water remaining after washing was blotted away. Then, the numbers of apoptotic cells and all cells were counted via optical microscopy after hematoxylin staining. The apoptosis rate was calculated as follows: apoptosis rate $=$ the number of apoptotic cells/the number of all cells $\times 100 \%$.

\section{Enzyme-linked immunosorbent assay (ELISA)}

Protein concentrations of serum interleukin-1 (IL-1), IL-6, matrix metalloproteinase-9 (MMP-9) and C-reactive protein (CRP) were measured using an ELISA assay kit (purchased from Jingmei Corporation, Shenzhen, China) after adding standard serum to 10 wells in the ELISA kit (including 2 blank wells without sample or ELISA reagent) and determining the standard curve according to the standard serum dilution gradient. Samples were properly diluted and added to the wells for analysis. The kit was gently shaken, covered with the provided sealer and incubated at $37^{\circ} \mathrm{C}$ for $30 \mathrm{~min}$. The contents were then removed from the wells, and each well was filled with wash buffer and gently shaken for $30 \mathrm{~s}$. The procedure was repeated 5 times. Then, chromogenic reagents $\mathrm{A}$ and B (50 $\mu \mathrm{L}$ of each) were added to each well. The wells were gently shaken and incubated in the dark at $37^{\circ} \mathrm{C}$ for $15 \mathrm{~min}$. The optical density (OD) at $450 \mathrm{~nm}$ of the samples was read within 15 min after adding $50 \mu \mathrm{L}$ of stop buffer. The ELISA assay kit was purchased from Bio-Rad, Inc., Hercules, CA, USA.

Quantitative real-time polymerase chain reaction ( $q R T-P C R$ )

Aortic ECs were collected from rats, and total RNA was extracted using Trizol reagent. The OD of each sample at 260/280 $\mathrm{nm}$ was detected using ultraviolet (UV) spectrophotometry, and the RNA concentration was calculated. Then, 500 ng of total RNA was subjected to reverse transcription, which was performed according to the instructions of an A3500 reverse transcription kit (Promega Corp., Madison, Wisconsin, USA). The PCR primers (Table 1) were designed with Primer Premier 5.0 in accordance with the gene sequence published in GenBank and were synthesized by Sangon Biotech Co., LTD., (Shanghai, China). A two-step qRT-PCR (SYBR GREEN) assay was performed as follows: pre-denaturation at $95^{\circ} \mathrm{C}$ for $15 \mathrm{~min}$, which was followed by 40 cycles of denaturation at $95^{\circ} \mathrm{C}$ for $30 \mathrm{~s}$ and extension at $60^{\circ} \mathrm{C}$ for $1 \mathrm{~min}$. The PCR reaction system was as follows: $12.5 \mu \mathrm{L}$ Premix Ex Taq or SYBR Green Mix, $1 \mu \mathrm{L}$ forward primer, $1 \mu \mathrm{L}$ reverse primer, 1-4 $\mu \mathrm{L}$ DNA template and up to $25 \mu \mathrm{L}$ dd $_{2} \mathrm{O}$. Glyceraldehyde-3-phosphate dehydrogenase (GAPDH) was used as an internal reference, and the standard control group values was set at 1 . Then, the threshold cycle value (Ct, threshold of amplification curves) of each target gene was analyzed. The relative expression of each target gene was calculated as a relative quantification (RQ) $=2^{-\Delta \Delta C t}$. The RQ values were used for statistical analysis. The PCR instrument (iQ5) was purchased from Bio-Rad, Inc. (Hercules, CA, USA).

Table 1. Primer sequences for the qRT-PCR analysis. Note: qRT-PCR, quantitative real-time polymerase chain reaction; cANRIL, circular antisense non-coding RNA in the INK4 locus; GAPDH, glyceraldehyde-3-phosphate dehydrogenase

\begin{tabular}{lll}
\hline Primer & Forward $\left(5^{\prime}-3^{\prime}\right)$ & Reverse $\left(5^{\prime}-3^{\prime}\right)$ \\
\hline cANRIL & TGCTCTATCCGCCAATCAGG & GGGCCTCAGTGGCACATACC \\
U6 & CTCGCTTCGGCAGCACATA & GTGCAGGGTCCGAGCT \\
bcl-2 & CGACTTTGCAGAGATGTCCA & ATGCCGGTTCAGGTACTCAG \\
Bax & CGTGGTTGCCCTCTTCTACTTT & TGATCAGCTCGGGCACTTTA \\
caspase-3 & ATGGACAACAACGAAACCTC & TTAGTGATAAAAGTACAGTTCTT \\
GAPDH & TCACTCCCGCGCAGTGAGGTT & TTTCTATTCACTACACTAGGG \\
\hline
\end{tabular}

\section{KARGER}


Immunohistochemistry

Paraffin-embedded tissue sections were heated at $60^{\circ} \mathrm{C}$ for $1 \mathrm{~h}$ and then deparaffinized with xylene twice for 5 min each. A gradient of ethanol and distilled water was used to hydrate the specimens; then, the specimens were boiled for 15 min in citrate buffer (pH 6.0, provided by Zhongshan Biotechnology Co., Ltd. Beijing, China), and endogenous peroxidase was inactivated. Appropriately diluted primary antibodies (1:250 rabbit anti-mouse Bax, $1 \mu \mathrm{g} / \mathrm{mL}$ rabbit anti-mouse bcl-2 and 1:50 rabbit anti-mouse caspase-3, all purchased from Abcam Inc., Cambridge, MA, USA) and corresponding secondary antibodies (Abcam Inc., Cambridge, MA, USA) were added. Samples were subsequently stained with DAB (Zhongshan biotechnology Co., Ltd. Beijing, China) and hematoxylin. Then, the samples were sealed with gum arabic after being dehydrated in ethanol. PBS was used instead of the primary antibodies in the control experiment. Brown particles observed via optical microscopy in the cytosol or nucleus indicated positively stained cells. Data were discontinuously collected, with every third section analyzed, and 10 fields of view were randomly chosen for each section. The percentages of positive cells relative to all cells counted under a high-power objective were calculated and analyzed.

\section{Statistical analysis}

Data were analyzed using the Statistical Package for the Social Sciences (SPSS) 21.0 (SPSS Inc., Chicago, IL, USA). Categorical data are expressed as the mean \pm standard deviation ( $\bar{x} \pm s$ ), and differences among multiple groups were analyzed via one-way analysis of variance (ANOVA). Comparisons among multiple groups were analyzed using the least significant difference (LSD) $t$ test; $P<0.05$ was considered statistically significant.

\section{Results}

Comparisons of the serum lipid levels and AI values of rats in the five groups

Compared with the values in the control group, the serum levels of total CHOL, TGs and LDL and the AI values increased and serum HDL levels decreased in the model, empty vector, over-expressed cANRIL and low-expressed cANRIL groups. Compared with the values in the model and empty vector groups, the serum levels of total CHOL, TGs and LDL and the AI values increased in the over-expressed cANRIL group, while serum HDL levels in decreased. Serum levels of total CHOL, TGs and LDL and AI values decreased and HDL levels increased in the low-expressed cANRIL group compared with the values in the model and empty vector groups (all $P<0.05$ ) (Table 2, Fig. 1).

Fig. 1. The AI of rats in the five groups. Note: *, $P<0.05$ compared with the control group; $\# P<0.05$ compared with the model and empty vector groups; AI, atherogenic index; and cANRIL, circular antisense non-coding RNA in the INK4 locus.

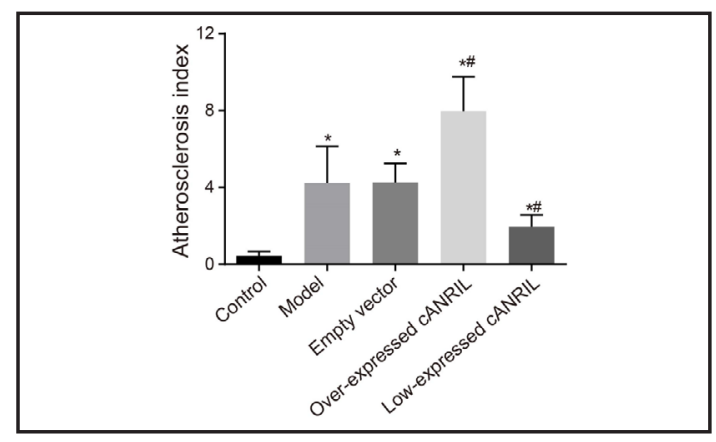

Table 2. Comparisons of serum lipid levels of the rats in the 5 groups. Note: ${ }^{*}, P<0.05$ compared with the control group; \#, $P<0.05$ compared with the model and empty vector \begin{tabular}{lllll}
\hline Group & $\mathrm{N}$ CHOL & TGs & LDL & HDL \\
$(\mathrm{mmol} / \mathrm{L})$ & $\begin{array}{l}(\mathrm{mmol} / \mathrm{L}) \\
(\mathrm{mmol} / \mathrm{L})\end{array}$ & \begin{tabular}{l}
$\mathrm{mmol} / \mathrm{L})$ \\
\hline
\end{tabular} \\
\hline
\end{tabular} groups; cANRIL, circular antisense Control $121.89 \pm 0.28 \quad 1.30 \pm 0.29 \quad 0.28 \pm 0.12 \quad 1.31 \pm 0.18$ non-coding RNA in the INK4 locus; Model $123.46 \pm 0.18^{*} \quad 2.34 \pm 0.68^{*} \quad 1.38 \pm 0.30^{*} \quad 0.66 \pm 0.22^{*}$ CHOL, cholesterol; TGs, triglycerides; Empty vector $123.42 \pm 0.68^{*} \quad 2.44 \pm 0.56^{*} \quad 1.37 \pm 0.31^{*} \quad 0.65 \pm 0.04^{*}$

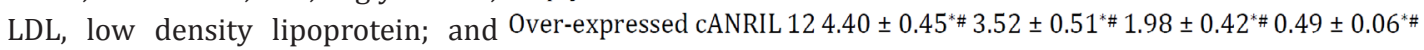
HDL, high density lipoprotein $\quad$ Low-expressed cANRIL $122.51 \pm 0.36^{*} 1.86 \pm 0.69^{*} 0.79 \pm 0.11^{*} 0.85 \pm 0.09^{*}$ 


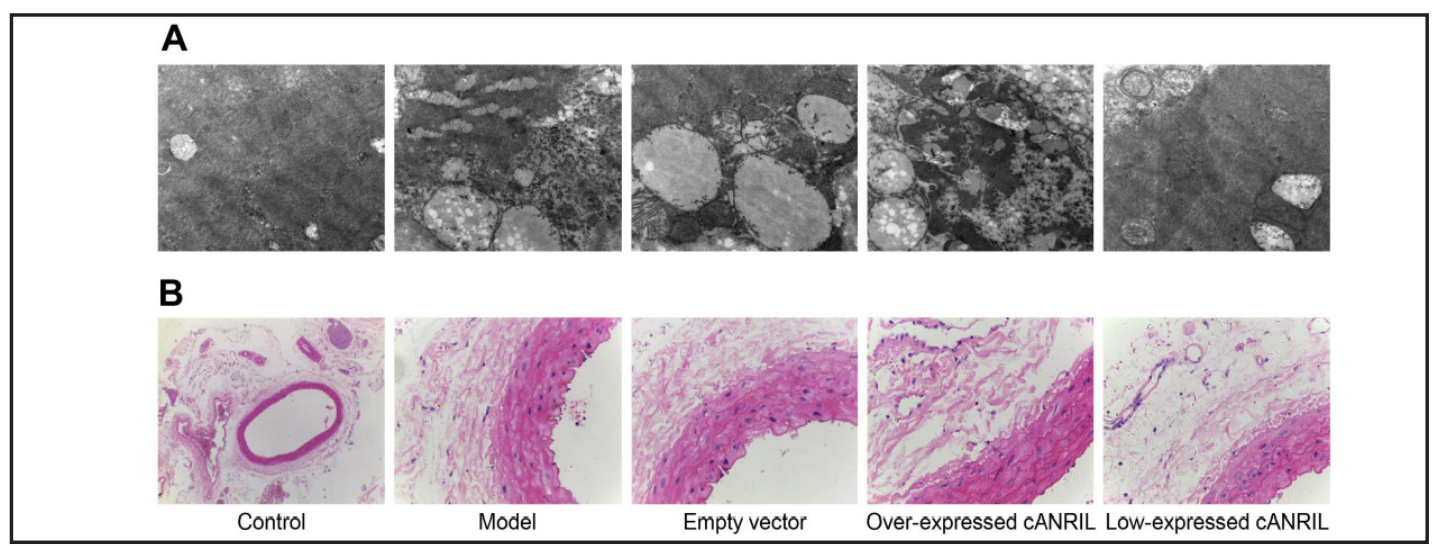

Fig. 2. Observation of ultrastructural and endothelial morphology of coronary arteries of rats in each group. Note: (A) shows a view of the ultrastructure of vascular ECs and SMCs from coronary arteries of rats in each group using an electron microscope $(\times 10000)$, and $(B)$ shows a view of the change in the endothelial morphology of coronary arteries of rats in each group observed using HE staining $(\times 200)$. EC, endothelial cell; SMC, smooth muscle cell; and cANRIL, circular antisense non-coding RNA in the INK4 locus.

Ultrastructural and endothelial morphology of coronary vessels in rats from the five groups

In the control group, rat ECs were closely arranged and spindle-shaped, all organelles had a normal structure without denaturation, and SMCs were arranged in parallel. In the model and empty vector groups, rat EC numbers were reduced, and the nuclei were irregular with nuclear pore expansion. Heterochromatin was gathered at the periphery of the nuclear membrane, and physalides were increasing observed in the cytoplasm. Additionally, the nuclei of SMCs were shrunken and their mitochondrion swollen. In the over-expressed cANRIL group, rat ECs were less abundant, and there was an increase in the number of physalides observed in the cytoplasm and in mitochondrial welling. However, in the lowexpressed cANRIL group, rat ECs were spindle-shaped with nuclei showing a normal structure, and SMCs were continuously and normally arranged (Fig. 2A). In accordance with the endothelial morphology observed via H\&E staining, the coronary arteries of rats in the control group were normal and did not show hyperplasia. In the model and empty vector groups, the coronary arteries of rats had disorganized structures, and visible atherosclerotic plaques and thrombi appeared. In the over-expressed cANRIL group, arterial structure was more disorganized and more thrombi appeared relative to the model and empty vector groups. The coronary arteries in the low-expressed cANRIL group had a normal structure, and some samples showed a tendency toward developing AS (Fig. 2B).

\section{Apoptosis of ECs in rats from the five groups}

TUNEL assay results showed that in the control group, only a few apoptotic ECs appeared in the coronary artery. The number of apoptosis-positive ECs showed a relative increase in the model, empty vector, over-expressed cANRIL and low-expressed cANRIL groups. Compared with the model and empty vector groups, the number of apoptotic ECs increased in the over-expressed cANRIL group, while it decreased in the low-expressed cANRIL group (all $P<0.05$ ) (Fig. 3).

Serum IL-1, IL-6, MMP-9 and CRP levels in rats from the five groups

ELISA results show that serum IL-1, IL-6, MMP-9 and CRP levels were increased in the model, empty vector, over-expressed cANRIL and low-expressed cANRIL groups compared with levels in the control group. Additionally, these levels were increased in the overexpressed cANRIL group and decreased in the low-expressed cANRIL group relative to levels in the model and empty vector groups (all $P<0.05$ ) (Table 3 ). 
Table 3. Serum levels of IL-1, IL-6, MMP-9 and CRP of rats in each group $(\mathrm{n}=12)$. Note: *, $P<0.05$ compared with the control group; \#, $P<0.05$ compared with the model and empty vector groups; cANRIL, circular antisense non-coding RNA in the INK4 locus; IL, interleukin; MMP-9, matrix metalloproteinase-9; and CRP, C-reactive protein

\begin{tabular}{lllll}
\hline Group & $\begin{array}{l}\mathrm{IL}-1 \\
(\mathrm{ng} / \mathrm{L})\end{array}$ & $\begin{array}{l}\mathrm{IL}-6 \\
(\mathrm{ng} / \mathrm{L})\end{array}$ & $\begin{array}{l}\text { MMP-9 } \\
(\mathrm{ng} / \mathrm{mL})\end{array}$ & $\begin{array}{l}\mathrm{CRP} \\
(\mathrm{pg} / \mathrm{mL})\end{array}$ \\
\hline Control & $185.34 \pm 31.42$ & $66.31 \pm 13.39$ & $53.79 \pm 10.34$ & $137.34 \pm 16.62$ \\
Model & $326.95 \pm 31.29^{*}$ & $256.42 \pm 25.78^{*}$ & $120.81 \pm 28.54^{*}$ & $199.62 \pm 17.21^{*}$ \\
Empty vector & $327.42 \pm 29.56^{*}$ & $268.43 \pm 27.65^{*}$ & $123.91 \pm 28.77^{*}$ & $197.36 \pm 11.74^{*}$ \\
Over-expressed cANRIL & $599.86 \pm 46.22^{*}$ & $392.76 \pm 35.37^{* \#}$ & $162.15 \pm 31.33^{* \#}$ & $243.87 \pm 16.04^{* \#}$ \\
Low-expressed cANRIL & $277.11 \pm 30.21^{* \#}$ & $148.96 \pm 14.32^{* \#}$ & $84.56 \pm 17.42^{* \#}$ & $166.64 \pm 12.13^{* \#}$ \\
\hline
\end{tabular}

Fig. 3. Over expression of cANRIL increased EC apoptosis in the coronary arteries of rats. Note: The black arrows indicate apoptotic cells (brown); the white arrows indicate non-apoptotic cells (blue). ${ }^{*}, P<$ 0.05 compared with the control group; \#, $P<$ 0.05 compared with the model and empty vector groups; EC, endothelial cell; and cANRIL, circular antisense non-coding RNA in the INK4 locus.

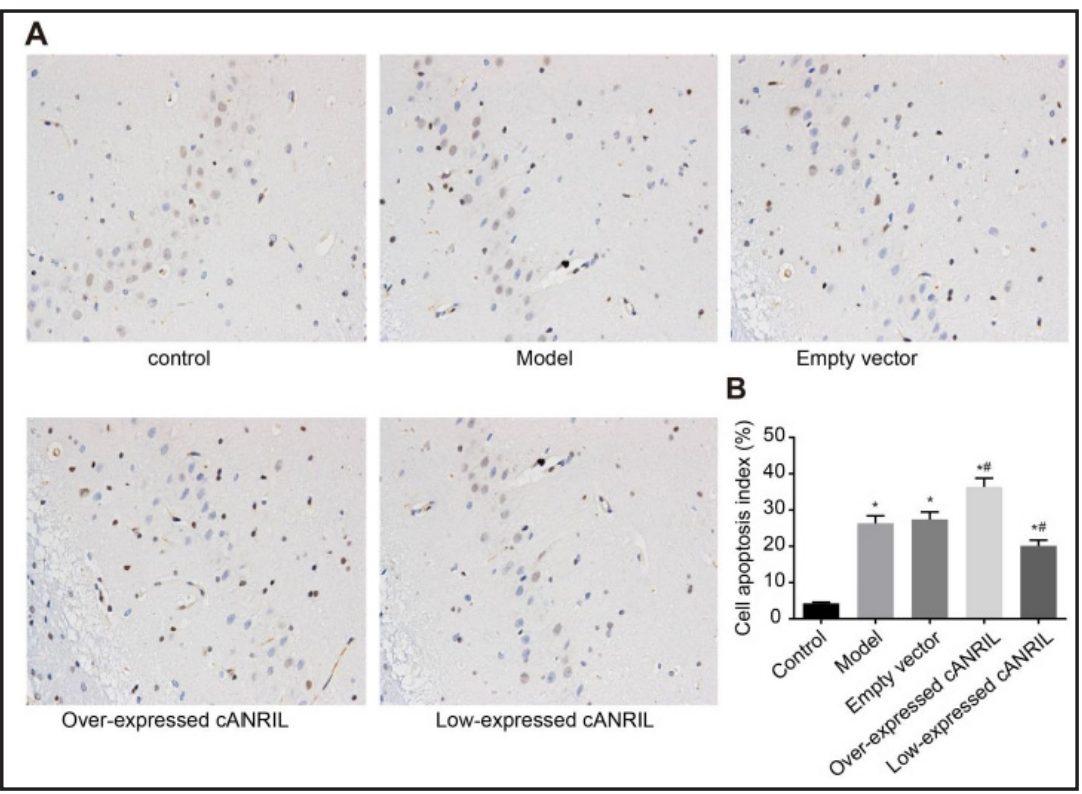

Fig. 4. The level of cANRIL and the mRNA expression levels of Bax, bcl-2 and caspase- 3 in ECs from rats in the five groups. Note: *, $P<0.05$ compared with the control group; \#, $P<0.05$ compared with the model and empty vector groups; cANRIL, circular antisense non-coding RNA in the INK4 locus; and EC, endothelial cell.

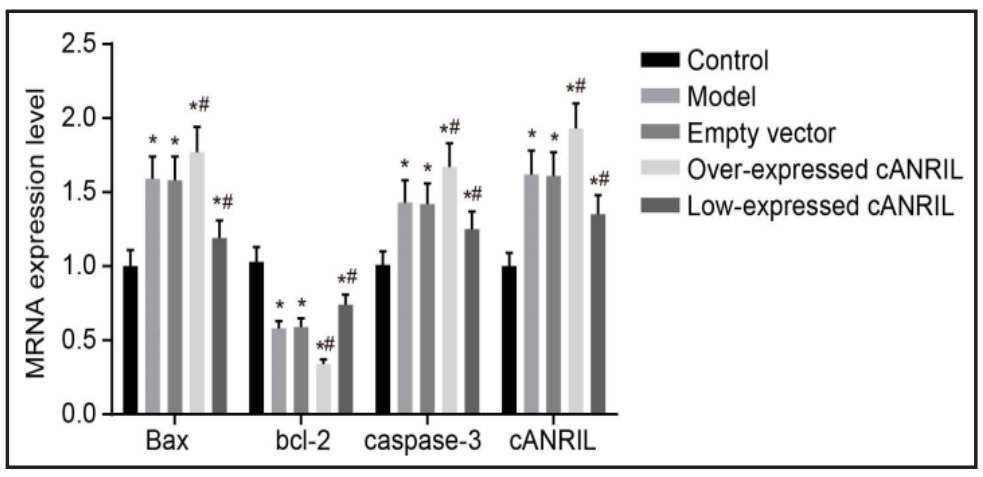

The mRNA expression of cANRIL, Bax, caspase-3 and bcl-2 in rats from the five groups

The qRT-PCR results demonstrate that compared with levels in the control group, the mRNA expression levels of Bax and caspase-3 increased and bcl-2 expression decreased in the model, empty vector, over-expressed cANRIL and low-expressed cANRIL groups. Additionally, compared with levels in the model and empty vector groups, mRNA expression levels of Bax and caspase-3 increased in the over-expressed cANRIL group and decreased in the low-expressed cANRIL group; an opposite trend was found bcl-2 mRNA expression in the low-expressed cANRIL group (all $P<0.05$ ) (Fig. 4).

\section{KARGER}


Fig. 5. Protein expression levels of Bax, bcl-2 and caspase-3 in ECs from rats in the five groups. Note: (A) shows the protein expression levels of Bax, bcl-2 and caspase-3 in ECs from each group; (B) shows the rates of positive cell staining for Bax, bcl-2, and caspase- 3 in the ECs from each group. *, $P<0.05$ compared with the control group; \#, $P<0.05$ compared with the model and empty vector groups; EC, endothelial cell; and cANRIL, circular antisense non-coding RNA in the INK4 locus.

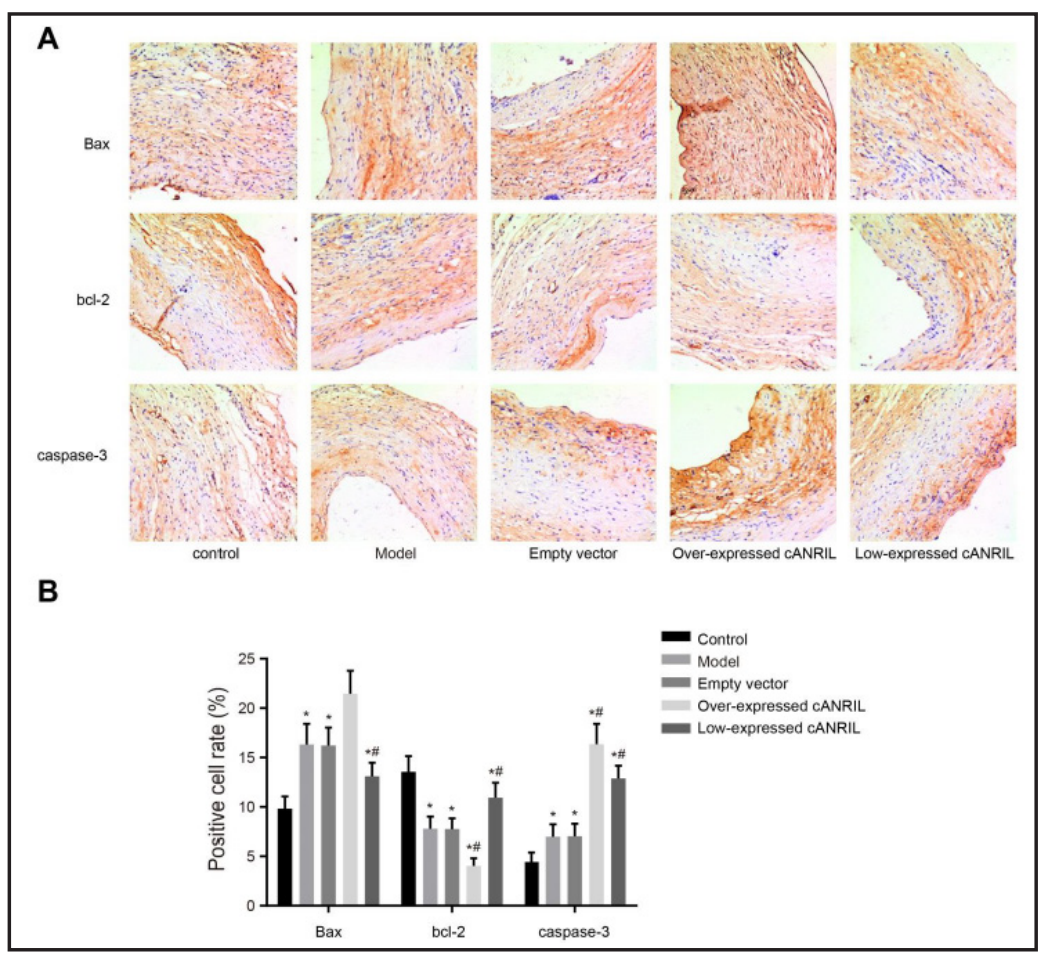

The protein expression of Bax, caspase-3 and bcl-2 in rats from the five groups

Immunohistochemistry results show that compared with levels in the control group, the protein expression levels of Bax and caspase-3 increased and bcl-2 expression decreased in rats in the model, empty vector, over-expressed cANRIL and low-expressed cANRIL groups. Compared with levels in the model and empty vector groups, the protein expression levels of Bax and caspase- 3 increased and bcl-2 expression decreased in the over-expressed cANRIL group. In the low-expressed cANRIL group, the protein expression levels of Bax and caspase-3 decreased and bcl-2 expression increased (all $P<0.05$ ) (Fig. 5).

\section{Discussion}

AS is the leading cause of stroke, CAD and vascular disease, and it predominantly contributes to morbidity and mortality worldwide [18]. ECs serve as a regulator of lipid transport, and inflammatory signal transduction and EC injury results in dysfunction that has been implicated in vascular inflammation and the development of AS [19]. Our study demonstrates that the expression of cANRIL was correlated with the expression of inflammatory factors in vascular ECs, and the over-expression of cANRIL could exacerbate vascular EC inflammation and promote AS.

Comparisons of serum lipid levels and AI values of rats in the five groups studied here indicated that elevated cANRIL expression could enhance changes in serum lipid levels and increase the AI. Skrzep-Poloczek et al. demonstrated that AS patients had an increased level of total CHOL and LDL and decreased level of HDL [20]. Chen et al. showed that AS patients had elevated levels of total CHOL, LDL and TGs [21]. In rats with AS, the ECs were irregular; for example, the number if ECs vanished, and nuclei were irregular with nuclear pore expansion, while heterochromatin gathered at the periphery of the nuclear membrane and physalides increased in the cytoplasm. AS is preferentially localized at bifurcations and bends in arterial trees, where irregular flow easily occurs. Now, it is well known that regular flow helps maintain vascular homeostasis, while irregular flow contributes to unfavorable vascular responses, which can eventually lead to vascular diseases [22, 23]. Additionally, our study found that elevated cANRIL expression could increase the levels of inflammatory 
factors, while reduced cANRIL expression could reduce the levels of inflammatory factors. Previous studies have shown that IL-1 could promote AS, and IL-6 levels are independently associated with subclinical AS [24, 25]. Markolf et al. showed that both hs-CRP and MMP9 are markers of inflammation released from macrophages, which contribute to unstable plaques and vascular remodeling [26].

Our study demonstrated that elevated cANRIL expression could lead to worse EC inflammation, exacerbating AS. cANRIL, which serves as a factor in atherosclerotic cardiovascular disease and is located on chromosome 9p21, induces cell apoptosis, inhibits cell proliferation and reduces atheroprotective effects [13]. Moreover, elevated cANRIL expression increased EC apoptosis in the coronary arteries of rats, but reduced cANRIL expression resulted in an opposite trend. These were consistent with the qRT-PCR and immunohistochemistry results as increased levels of apoptosis-related genes and proteins was observed in the over-expressed cANRIL group. Vascular cell apoptosis, a form of cell death, plays a significant role in the development of AS, which promotes cardiovascular events [27]. Increases in apoptosis may lead to endothelial dysfunction, which is a key event in the progression of AS [28, 29]. George et al. revealed that the increased expression of Bax and caspase resulted in apoptosis [30]. Additionally, Xu et al. demonstrated that elevated bcl-2 expression could prevent apoptosis, blocking the apoptosis induced by several agents in several cell types, including ECs [31].

In summary, our study provides evidence that elevated cANRIL expression could promote AS by exacerbating the inflammation of ECs and increasing the serum levels of lipids, CHOL, TGs, LDL, IL-1, IL-6, MMP-9 and CRP. cANRIL plays a crucial role in aggravating vascular EC inflammation in AS. Additionally, this study strongly suggests that reduced cANRIL expression might have a protective effect in the therapeutic treatment of patients with AS. We also realize that in order to fully clarify the direct mechanism of action of cANRIL in AS, further experimental investigations and clinical trials are needed. Nonetheless, the data from this study could shed light on a potentially new, targeted method to prevent AS using an RNA-based approach.

\section{Acknowledgments}

This project was supported by the Jilin Provincial Education Department's "12th FiveYear" Scientific and Technological Research Projects, Ji Zi UNESCO ([2014]B016), the Young Scientific and Technological Innovation Leading Talent and Team Projects of Jilin Provincial Science and Technology Department (20150519025JH), the Development and Reform Commission Industrial Technology Research and Development Project of Jilin Province (2015Y030-3), the Education Department "12th Five-Year" Industrialization Cultivation Planning Project of Jilin Province ([2015]524), and the Development and Reform Commission Provincial Industrial Innovation Special Fund Project (High-tech Industry Part) Industrial Technology Research and Development Projects of Jilin Province (2016C044-2). We would also like to thank all the participants who helped conduct the present study.

\section{Disclosure Statement}

We declare that we have no conflicts of interest.

\section{References}

1 Libby P, Ridker PM, Hansson GK: Progress and challenges in translating the biology of atherosclerosis. Nature 2011;473:317-325.

2 Weber C, Noels H: Atherosclerosis: current pathogenesis and therapeutic options. Nat Med 2011;17:14101422. 


\section{Cellular Physiology Cell Physiol Biochem 2017;42:1202-1212 \begin{tabular}{l|l} 
and Biochemistry Published onlıne: July 03, 2017 & $\begin{array}{l}\text { (c) } 2017 \text { The Author(s). Published by S. Karger AG, Basel } \\
\text { www.karger.com/cpb }\end{array}$
\end{tabular}}

Song et al.: Role of cANRIL in Atherosclerosis

3 Buttari B, Profumo E, Rigano R: Crosstalk between red blood cells and the immune system and its impact on atherosclerosis. Biomed Res Int 2015;2015:616834.

4 Moore KJ, Tabas I: Macrophages in the pathogenesis of atherosclerosis. Cell 2011;145:341-355.

5 Maier JA: Endothelial cells and magnesium: implications in atherosclerosis. Clin Sci (Lond) 2012;122:397407.

-6 Onat D, Brillon D, Colombo PC, Schmidt AM: Human vascular endothelial cells: a model system for studying vascular inflammation in diabetes and atherosclerosis. Curr Diab Rep 2011;11:193-202.

7 Gimbrone MA, Jr., Garcia-Cardena G: Endothelial Cell Dysfunction and the Pathobiology of Atherosclerosis. Circ Res 2016;118:620-636.

-8 Sun X, He S, Wara AK, Icli B, Shvartz E, Tesmenitsky Y, Belkin N, Li D, Blackwell TS, Sukhova GK, Croce K, Feinberg MW: Systemic delivery of microRNA-181b inhibits nuclear factor-kappaB activation, vascular inflammation, and atherosclerosis in apolipoprotein E-deficient mice. Circ Res 2014;114:32-40.

-9hang W, Chen Y, Liu P, Chen J, Song L, Tang Y, Wang Y, Liu J, Hu FB, Hui R: Variants on chromosome 9p21.3 correlated with ANRIL expression contribute to stroke risk and recurrence in a large prospective stroke population. Stroke 2012;43:14-21.

10 Kral BG, Mathias RA, Suktitipat B, Ruczinski I, Vaidya D, Yanek LR, Quyyumi AA, Patel RS, Zafari AM, Vaccarino V, Hauser ER, Kraus WE, Becker LC, Becker DM: A common variant in the CDKN2B gene on chromosome 9p21 protects against coronary artery disease in Americans of African ancestry. J Hum Genet 2011;56:224-229.

11 Bochenek G, Hasler R, El Mokhtari NE, Konig IR, Loos BG, Jepsen S, Rosenstiel P, Schreiber S, Schaefer AS: The large non-coding RNA ANRIL, which is associated with atherosclerosis, periodontitis and several forms of cancer, regulates ADIPOR1, VAMP3 and C110RF10. Hum Mol Genet 2013;22:4516-4527.

12 Schaefer AS, Richter GM, Groessner-Schreiber B, Noack B, Nothnagel M, El Mokhtari NE, Loos BG, Jepsen S, Schreiber S: Identification of a shared genetic susceptibility locus for coronary heart disease and periodontitis. PLoS Genet 2009;5:e1000378.

-13 Holdt LM, Stahringer A, Sass K, Pichler G, Kulak NA, Wilfert W, Kohlmaier A, Herbst A, Northoff BH, Nicolaou A, Gabel G, Beutner F, Scholz M, Thiery J, Musunuru K, Krohn K, Mann M, Teupser D: Circular noncoding RNA ANRIL modulates ribosomal RNA maturation and atherosclerosis in humans. Nat Commun 2016;7:12429.

14 Qian Y, Lu Y, Rui C, Qian Y, Cai M, Jia R: Potential Significance of Circular RNA in Human Placental Tissue for Patients with Preeclampsia. Cell Physiol Biochem 2016;39:1380-1390.

15 Li H, Hao X, Wang H, Liu Z, He Y, Pu M, Zhang H, Yu H, Duan J, Qu S: Circular RNA Expression Profile of Pancreatic Ductal Adenocarcinoma Revealed by Microarray. Cell Physiol Biochem 2016;40:1334-1344.

16 Wu HJ, Zhang CY, Zhang S, Chang M, Wang HY: Microarray Expression Profile of Circular RNAs in Heart Tissue of Mice with Myocardial Infarction-Induced Heart Failure. Cell Physiol Biochem 2016;39:205-216.

17 Maiese K: Disease onset and aging in the world of circular RNAs. J Transl Sci 2016;2:327-329.

18 Charo IF, Taub R: Anti-inflammatory therapeutics for the treatment of atherosclerosis. Nat Rev Drug Discov 2011;10:365-376.

19 Pollreisz A, Hudson BI, Chang JS, Qu W, Cheng B, Papapanou PN, Schmidt AM, Lalla E: Receptor for advanced glycation endproducts mediates pro-atherogenic responses to periodontal infection in vascular endothelial cells. Atherosclerosis 2010;212:451-456.

20 Skrzep-Poloczek B, Tomasik A, Tarnawski R, Hyla-Klekot L, Dyduch A, Wojciechowska C, Wesolowski W, Kopieczna-Grzebieniak E, Zalejska-Fiolka J, Widera E: Nephrotic origin hyperlipidemia, relative reduction of vitamin E level and subsequent oxidative stress may promote atherosclerosis. Nephron 2001;89:68-72.

21 Chen G, Cai L, Chen B, Liang J, Lin F, Li L, Lin L, Yao J, Wen J, Huang H: Serum level of endogenous secretory receptor for advanced glycation end products and other factors in type 2 diabetic patients with mild cognitive impairment. Diabetes Care 2011;34:2586-2590.

22 Hsieh HJ, Liu CA, Huang B, Tseng AH, Wang DL: Shear-induced endothelial mechanotransduction: the interplay between reactive oxygen species (ROS) and nitric oxide (NO) and the pathophysiological implications. J Biomed Sci 2014;21:3.

23 VanderLaan PA, Reardon CA, Getz GS: Site specificity of atherosclerosis: site-selective responses to atherosclerotic modulators. Arterioscler Thromb Vasc Biol 2004;24:12-22. 


\section{Cellular Physiology Cell Physiol Biochem 2017;42:1202-1212 \begin{tabular}{l|l} 
DOI: 10.1159/000478918 & $\begin{array}{l}\text { O 2017 The Author(s). Published by S. Karger AG, Basel } \\
\text { www.karger.com/cpb }\end{array}$
\end{tabular}}

Song et al.: Role of cANRIL in Atherosclerosis

24 Alexander MR, Moehle CW, Johnson JL, Yang Z, Lee JK, Jackson CL, Owens GK: Genetic inactivation of IL-1 signaling enhances atherosclerotic plaque instability and reduces outward vessel remodeling in advanced atherosclerosis in mice. J Clin Invest 2012;122:70-79.

25 Amar J, Fauvel J, Drouet L, Ruidavets JB, Perret B, Chamontin B, Boccalon H, Ferrieres J: Interleukin 6 is associated with subclinical atherosclerosis: a link with soluble intercellular adhesion molecule 1. J Hypertens 2006;24:1083-1088.

-26 Hanefeld M, Pfutzner A, Forst T, Kleine I, Fuchs W: Double-blind, randomized, multicentre, and active comparator controlled investigation of the effect of pioglitazone, metformin, and the combination of both on cardiovascular risk in patients with type 2 diabetes receiving stable basal insulin therapy: the PIOCOMB study. Cardiovasc Diabetol 2011;10:65.

-27 Katsiki N, Tziomalos K, Chatzizisis Y, Elisaf M, Hatzitolios AI: Effect of HMG-CoA reductase inhibitors on vascular cell apoptosis: beneficial or detrimental? Atherosclerosis 2010;211:9-14.

28 Wang P, Xu TY, Guan YF, Zhao Y, Li ZY, Lan XH, Wang X, Yang PY, Kang ZM, Vanhoutte PM, Miao CY: Vascular smooth muscle cell apoptosis is an early trigger for hypothyroid atherosclerosis. Cardiovasc Res 2014;102:448-459.

29 Qin W, Ren B, Wang S, Liang S, He B, Shi X, Wang L, Liang J, Wu F: Apigenin and naringenin ameliorate PKCbetaII-associated endothelial dysfunction via regulating ROS/caspase-3 and NO pathway in endothelial cells exposed to high glucose. Vascul Pharmacol 2016;85:39-49.

-30 George J, Gondi CS, Dinh DH, Gujrati M, Rao JS: Restoration of tissue factor pathway inhibitor-2 in a human glioblastoma cell line triggers caspase-mediated pathway and apoptosis. Clin Cancer Res 2007;13:35073517.

-31 Xu G, Gong Z, Yu W, Gao L, He S, Qian Z: Increased expression ratio of Bcl-2/Bax is associated with crocinmediated apoptosis in bovine aortic endothelial cells. Basic Clin Pharmacol Toxicol 2007;100:31-35. 